\title{
Hepatobiliary Neoplasm
}

National Cancer Institute

\section{Source}

National Cancer Institute. Hepatobiliary Neoplasm. NCI Thesaurus. Code C8614.

A benign or malignant neoplasm that affects the liver parenchyma, bile ducts, and gallbladder. Representative examples of benign neoplasms include hepatocellular adenoma, bile duct adenoma, and gallbladder lipoma. Representative examples of malignant neoplasms include hepatocellular carcinoma, intrahepatic and extrahepatic cholangiocarcinoma, and gallbladder carcinoma. 